\title{
Regions in the nonstandard plane that contain no standard points
}

\author{
STEVEN C. LETH
}

\begin{abstract}
Nonstandard methods are applied to continuum theory in the plane. The main result concerns internal regions $V$ that contain no standard points and have the property that $s t(V)$ does not disconnect the plane and are bounded by simple closed curves that have small intersection with ${ }^{*} s t(V)$. We show that such sets cannot contain a "Y-set" consisting of three arcs intersecting only at a single common point with the property that the non-intersecting endpoints of each arc are a noninfinitesimal distance from the arc joining the other two ends.
\end{abstract}

2010 Mathematics Subject Classification 54J05, 54F15 (primary); 03H05 (secondary)

Keywords: nonstandard topology, continuum theory

\section{Preliminaries}

A continuum is a compact, connected metric space. Here we are concerned solely with continua in the plane and the nonstandard plane. A good general reference for Continuum Theory is [15]. A short historical perspective is given in [8].

We work in a nonstandard universe, as described in detail for example in [3],[5],[10], or [11]. We assume that our universe is $\beth_{1}^{+}$saturated, as this is needed in some of the results.

Definition 1.1 If $p_{1}$ and $p_{2}$ are points in the nonstandard plane we will write $p_{1} \approx p_{2}$ if they are an infinitesimal distance apart and we will say that $p_{1}$ is near $p_{2}$.

If $A$ is a standard set we will write ${ }^{*} \mathbf{A}$ for its nonstandard counterpart.

If $p$ is a point in the nonstandard plane that is within some standard distance of the origin we write st(p) for the unique standard point that is within an infinitesimal distance of $p$.

The elements of ${ }^{*} P\left(\mathbb{R}^{2}\right)$ are the internal sets (here we use $P(A)$ to denote the power set of $A$ ). Intuitively these are the subsets of $* \mathbb{R}^{2}$ that the nonstandard model recognizes as sets. 
The results in the proposition below are elementary and well-known, but are fundamental to this work, so the proofs are included here for convenience.

Proposition 1.2 i) If $A$ is any internal set in the plane then st $(A)$ (the set of all standard points infinitesimally close to points in A) is closed.

ii) If $A$ is internal, connected and bounded (i.e. contained in some ${ }^{*} B(0, r)$ for a standard real $r>0)$ in the nonstandard plane then $s t(A)$ is connected.

Proof i) If $p$ is a limit point of $\operatorname{st}(A)$ then for any standard $\varepsilon>0$ there must exist points of $A$ within $\varepsilon$ of $p$. Internally we may let $r=\inf _{a \in A}\|a-p\|$, and $r$ must be infinitesimal. But then $p$ is a standard point near points in $A$, so $p \in \operatorname{st}(A)$.

ii). Suppose that $\operatorname{st}(A)$ is not connected, and let $U$ and $V$ be a separation, i.e. suppose that $s t(A) \subset U \cup V$, both are open, and both have nonempty intersection with $s t(A)$. Let $u \in U \cap s t(A)$ and $v \in V \cap s t(A)$. Then there exists $a_{u}, a_{v}$ in $A$ such that $\operatorname{st}\left(a_{u}\right)=u$ and $s t\left(a_{v}\right)=v$. Since $U$ and $V$ are open, there exists a standard $d>0$ such that all points within $d$ of $u$ are in $U$ and all points within $d$ of $v$ are in $V$. Thus, by transfer, $a_{u} \in{ }^{*} U$, and $a_{v} \in{ }^{*} V$, and these two sets are open and disjoint. Since $A$ is connected and both ${ }^{*} U$ and ${ }^{*} V$ intersect $A$, there must exist a point $x \in A$ that is not contained in ${ }^{*} U \cup{ }^{*} V$. Since $A$ is bounded $s t(x)$ exists and since $s t(x)$ is an element of $\operatorname{st}(A)$, it is contained in either $U$ or $V$. As above, however, this means that $x$ is in either ${ }^{*} U$ or ${ }^{*} V$, and this contradiction completes the proof.

Most of the definitions needed here are all collected below. The two "Y-set" definitions do not seem to correspond exactly to other definitions in the literature. They are simple triods with appropriate "width" properties. The notion of "IL-chainable" is a natural extension of usual chainability definitions into this nonstandard setting.

Definition 1.3 If $A$ is an arc (either standard or internal) and $a_{1}$ and $a_{2}$ are two points on $A$ then we will write $\mathbf{A}\left[\mathbf{a}_{1}, \mathbf{a}_{2}\right]$ for the subarc from $a_{1}$ to $a_{2}$. If $S$ is a simple closed curve in the plane or the nonstandard plane we will write $\mathbf{V}_{S}$ for the bounded region whose boundary is $S$ ( $V_{S}$ exists and is well-defined by the Jordan Curve Theorem). We will say that a set $A \subset{ }^{*} \mathbb{R}^{2}$ contains a Y-set if there exist four points $a, b, c$, and $x$ in $A$, arcs $C_{a x}, C_{b x}$, and $C_{c x}$ in $A$ intersecting only at $x$, from $a$ to $x, b$ to $x$, and $c$ to $x$, respectively, such that none of the points $a, b$, or $c$ are within an infinitesimal of any point on the arcs joining the others to $x$ (thus, for example, no point of $C_{a x}$ 
is infinitesimally close to $b$ or to $c$ ). If $\delta>0$ we will say that a set $A$ in the plane contains a size $\delta$ Y-set if there exist four points $a, b, c$, and $x$ in $A$, and $\operatorname{arcs} C_{a x}, C_{b x}$, and $C_{c x}$ in $A$ intersecting only at $x$ from $a$ to $x, b$ to $x$, and $c$ to $x$, respectively, such that none of the points $a, b$, or $c$ are within $\delta$ of any point on the arcs joining the others to $x$ (thus, for example, no point of $C_{a x}$ is within distance $\delta$ of $b$ or $c$ ). We will call an internal subset of the nonstandard plane IL-chainable (for "infinitesimally linearly chainable") if it can be covered by a hyperfinite collection of open sets $G_{0}, \ldots, G_{n}$ of infinitesimal diameter with the "linear chain" condition that $G_{i} \cap G_{j}$ is nonempty iff $|i-j| \leq 1$.

A standard set that can be covered by a finite chain of sets $G_{i}$ as in the above definition is called $\delta$-chainable if each of the chaining sets are of diameter less than $\delta$. A set is called chainable if it is $\delta$-chainable for all $\delta>0$. So, IL-chainable sets are just those that satisfy the usual definition of being $\delta$-chainable inside the nonstandard model for some infinitesimal $\delta>0$.

\section{Regions Containing No Standard Points}

In this section we exploit the interplay between a set and the internal set obtained by first taking the standard part and then looking at the nonstandard version of that set. Of particular importance are regions $V$ that have little intersection with ${ }^{*} s t(V)$ and that contain no standard points. It is easy to find simple examples of regions $V$ such that ${ }^{*} s t(V) \cap V$ is empty. For example if $\eta>0$ is infinitesimal and $V$ is the interior of the rectangle with corners at $(\eta, 1),(2 \eta, 1),(2 \eta, 0)$ and $(\eta, 0)$ then $s t(V)$ is simply the closed line segment on the $y$ axis from $(0,0)$ to $(0,1)$. The set ${ }^{*} s t(V)$ is the nonstandard counterpart of $s t(V)$, so includes nonstandard points on this line segment, including points within an infinitesimal of the origin, but since the standard universe satisfies the statement that all the points in $s t(V)$ have $x$ coordinate of 0 the nonstandard version of this set has the same property by the transfer principle. This shows that ${ }^{*} \operatorname{st}(V) \cap V$ is empty. The boundary of $V$ does not intersect ${ }^{*} s t(V)$ at all in this example. However in the most interesting cases, such as regions obtained by blocking off long portions of a simple dense canal, there will always be some intersection with * $s t(V)$, although only on the small segments used to close off a region. There will always be many points of ${ }^{*} s t(V)$ in the interior of $V$, but the crucial additional condition in the theorem below is that none of them will be actual standard points. 
Theorem 2.1 Let $V \subset{ }^{*} \mathbb{R}^{2}$ be a bounded (internal) region (i.e. contained in some ${ }^{*} B(0, r)$ for a standard real $\left.r>0\right)$ bounded by a simple closed curve $S$ and suppose that $\bar{V}$ contains no standard points, st $(V)$ does not disconnect the plane, and that there exists an arc $A$ of infinitesimal length on $S$ such that ${ }^{*} s t(V) \cap S \subset A$. Then $V$ contains no Y-set.

Proof We first note that $s t(V)$ has no interior. Since $\bar{V}$ contains no standard points, every point of ${ }^{*} s t(V)$ is near a point of its boundary $S$. If ${ }^{*} s t(V)$ were to contain any ball of noninfinitesimal diameter, then it would intersect $S$ at points that are not all near each other, which violates the condition that ${ }^{*} s t(V) \cap S \subset A$. Thus, $s t(V)$ has no interior by transfer.

Suppose that $V$ contains a Y-set. We let $a, b, c$ and $x$ be points as in the definition of a Y-set, but we note that there are many possible choices for $a, b$, and $c$, and since the $\operatorname{arc} A$ is of infinitesimal length, we may choose these points in such a way that none of them are near $A$. We let $Y=C_{a x} \cup C_{b x} \cup C_{c x}$.

We next show that there must exist standard disjoint balls $B_{a}, B_{b}$, and $B_{c}$ satisfying the following:

i) $a \in{ }^{*} B_{a}, b \in{ }^{*} B_{b}$, and $c \in{ }^{*} B_{c}$.

ii) Each of these three balls have non-zero radius (and thus non-infinitesimal radius since they are standard sets).

iii) The arc $A$ does not intersect $\overline{B_{a}}, \overline{B_{b}}$, or $\overline{B_{c}}$.

iv) every point on $\overline{B_{a}}$ is not near any point on $C_{b x}$ or $C_{c x}$, every point on $\overline{B_{b}}$ is not near any point on $C_{a x}$ or $C_{c x}$, and every point on $\overline{B_{c}}$ is not near any point on $C_{a x}$ or $C_{b x}$.

v) The interior of each ball contains points not disconnected from infinity by $\operatorname{st}(V)$ together with the closure of the other two balls. For example, the interior of $B_{a}$ contains points not disconnected from infinity by $s t(V) \cup \overline{B_{b}} \cup \overline{B_{c}}$.

It is clear from the conditions given that standard balls of sufficiently small radius can be found that will satisfy the first four properties, and we assume that we begin with a $B_{a}^{0}, B_{b}^{0}$, and $B_{c}^{0}$ satisfying just those. It is possible that, for example, $s t(V) \cup \overline{B_{b}^{0}} \cup \overline{B_{c}^{0}}$ disconnects the plane. However, since $s t(V)$ does not disconnect the plane, and no point of $\overline{B_{b}^{0}}$ is near $\overline{B_{c}^{0}}$ or any point on $C_{a x}$ or $C_{c x}$, a bounded component of the complement of $s t(V) \cup \overline{B_{b}^{0}} \cup \overline{B_{c}^{0}}$ could occur only if a portion of $C_{b x}$ encloses some standard open set and then passes back into or within an infinitesimal of $\overline{B_{b}^{0}}$ or if a portion of $C_{c x}$ encloses some standard open set and then passes back into or within 
an infinitesimal of $\overline{B_{c}^{0}}$. If $B_{a}^{0}$ is in such a bounded component (all points in this set must be in the same component since no point of the boundary intersects $B_{a}^{0}$ ) we let $p_{a}$ be a standard point in $B_{a}^{0}-s t(V)$, and $T_{a}$ be a standard path from $p_{a}$ to infinity in $\mathbb{R}^{2}-(s t(V))$. Such a path exists since $\mathbb{R}^{2}-(s t(V))$ is open and connected, and thus path-connected. This standard path cannot pass near $b$ or $c$ since it stays a standard nonzero distance away from $s t(V)$. Similarly if $B_{b}^{0}$ is in a bounded component of the complement of $s t(V) \cup \overline{B_{a}^{0}} \cup \overline{B_{c}^{0}}$ we let $p_{b}$ be a standard point in $B_{b}^{0}-s t(V)$, and $T_{b}$ be a standard path from $p_{a}$ to infinity in $\mathbb{R}^{2}-(s t(V))$, and if $B_{c}^{0}$ is in a bounded component of the complement of $s t(V) \cup \overline{B_{a}^{0}} \cup \overline{B_{b}^{0}}$ we let $p_{c}$ be a standard point in $B_{c}^{0}-s t(V)$, and $T_{c}$ be a standard path from $p_{c}$ to infinity in $\mathbb{R}^{2}-(s t(V))$. We can now choose smaller balls $B_{a}, B_{b}$, and $B_{c}$ inside $B_{a}^{0}, B_{b}^{0}$, and $B_{c}^{0}$ such that conditions i) through iv) are satisfied and none of these intersect $T_{a}, T_{b}$ or $T_{c}$. This ensures that condition v) will hold, for points in $\left(\mathbb{R}^{2}-(s t(V))\right) \cap B_{a}$ are in the same connected component of the complement of $s t(V) \cup \overline{B_{b}} \cup \overline{B_{c}}$ as $p_{a}$, points in $\left(\mathbb{R}^{2}-(s t(V))\right) \cap B_{b}$ are in the same connected component of the complement of $s t(V) \cup \overline{B_{a}} \cup \overline{B_{c}}$ as $p_{b}$ and points in $\left(\mathbb{R}^{2}-(s t(V))\right) \cap B_{c}$ are in the same connected component of the complement of $s t(V) \cup \overline{B_{a}} \cup \overline{B_{b}}$ as $p_{c}$.

Having established the existence of the standard balls satisfying conditions i) through v), we now let $a^{\prime}$ be the last point of $C_{a x} \cap \overline{B_{a}}$ on the arc from $a$ to $x$, and define $C_{a^{\prime} x}$ to be the subarc of $C_{a x}$ from $a^{\prime}$ to $x$. We define $b^{\prime}, C_{b^{\prime} x}, c^{\prime}, C_{c^{\prime} x}$ analogously. We let $Y^{\prime}=C_{a^{\prime} x} \cup C_{b^{\prime} x} \cup C_{c^{\prime} x}$, and we note that $Y^{\prime}$ is path-connected and therefore connected. By proposition $1 \operatorname{st}\left(Y^{\prime}\right)$ is connected, and thus ${ }^{*} s t\left(Y^{\prime}\right)$ is connected by transfer.

We let $q_{a}$ be a standard point in $B_{a}-s t(V)$ and $T_{a}^{\prime}$ be a standard infinite path (ray) in $\mathbb{R}^{2}-\left(s t(V) \cup \overline{B_{b}} \cup \overline{B_{c}}\right)$ starting at $q_{a}$ and going off to infinity. Similarly we let $q_{b}$ be a standard point in $B_{b}-s t(V)$ and $T_{b}^{\prime}$ be a standard infinite path in $\mathbb{R}^{2}-$ $\left(s t(V) \cup \overline{B_{b}} \cup \overline{B_{c}} \cup T_{a}^{\prime}\right.$ ) starting at $q_{b}$ and going off to infinity, and $q_{c}$ be a standard point in $B_{c}-s t(V)$ and $T_{c}^{\prime}$ be a standard infinite path (ray) in $\mathbb{R}^{2}-\left(s t(V) \cup \overline{B_{b}} \cup \overline{B_{c}} \cup T_{a}^{\prime} \cup T_{b}^{\prime}\right)$ starting at $q_{c}$ and going off to infinity. Figure 1 illustrates these definitions.

Since $s t(V)$ and any bounded portion of $T_{a}^{\prime}, T_{b}^{\prime}$, and $T_{c}^{\prime}$ are disjoint compact sets, they are some non-zero distance apart, so by transfer there are no points of ${ }^{*} s t(V)$ that are within an infinitesimal distance of any points of ${ }^{*} T_{a}^{\prime},{ }^{*} T_{b}^{\prime}$, or ${ }^{*} T_{c}^{\prime}$. We note that the single point $s t(A)$ is in $s t(V)$, so the arc $A$ is also not within an infinitesimal distance of any points of ${ }^{*} T_{a},{ }^{*} T_{b}$, or ${ }^{*} T_{c}$.

We now define internal infinite arcs as follows: We let $\Gamma_{a}$ consist of the line segment from $a^{\prime}$ to a nearest point of ${ }^{*} T_{a}^{\prime} \cap \overline{B_{a}}$, followed by ${ }^{*} T_{a}^{\prime}$ from that point to infinity. There may be more than one "nearest point" and we can choose any of them. This 


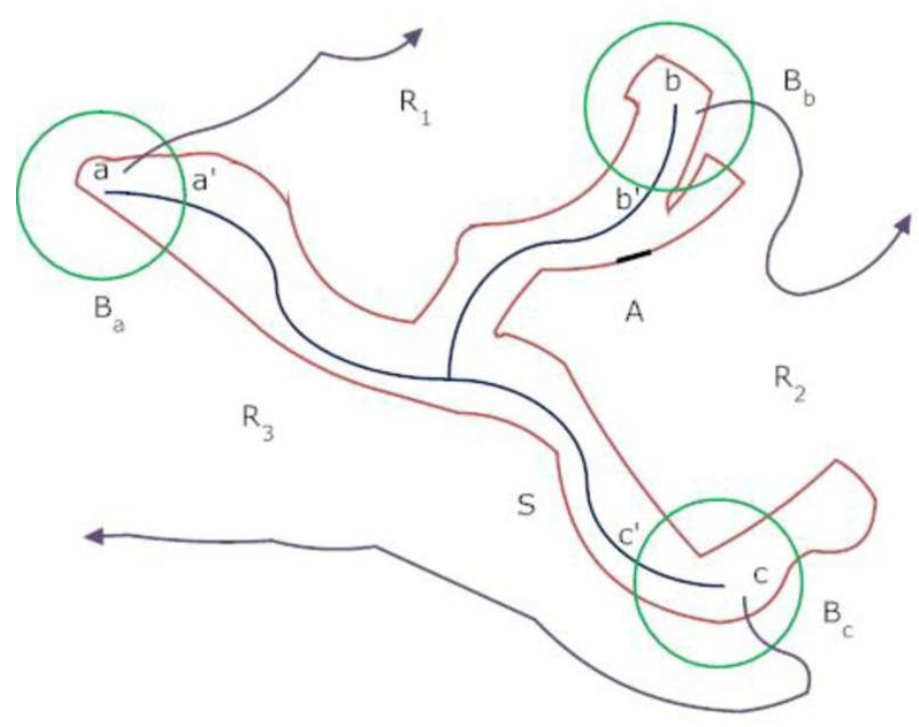

Figure 1: Illustration of definitions

condition ensures that the initial segment does not intersect ${ }^{*} T_{a}^{\prime}$ except at this point. The internal paths $\Gamma_{b}$ and $\Gamma_{c}$ are defined analogously. Since there are no points of ${ }^{*} s t\left(Y^{\prime}\right)$ in the interior of $B_{a}, B_{b}$, and $B_{c}$ there are no points of ${ }^{*} s t\left(Y^{\prime}\right)$ other than $a^{\prime}$ that intersect $\Gamma_{a}$, and similarly the other two arcs only intersect ${ }^{*} s t\left(Y^{\prime}\right)$ at their initial points. Since the $\operatorname{arc} A$ does not intersect $\overline{B_{a}}, \overline{B_{b}}$, or $\overline{B_{c}}$ we also know that $A$ does not intersect $\Gamma_{a}, \Gamma_{b}$, or $\Gamma_{c}$.

The set $\Gamma_{a} \cup \Gamma_{b} \cup \Gamma_{c} \cup Y^{\prime}$ consists of three non-intersecting infinite arcs originating from the point $x$, and so divides the nonstandard plane into three regions. One of these regions, which we will call $R_{1}$, is bounded by $\Gamma_{a} \cup C_{a^{\prime} x} \cup C_{b^{\prime} x} \cup \Gamma_{b}$. No point in this region can be within an infinitesimal distance of $c^{\prime}$, since $c^{\prime}$ is not contained in $R_{1}$ and is not near any of the points on the boundary. Similarly we let $R_{2}$ be the region bounded by $\Gamma_{b} \cup C_{b^{\prime} x} \cup C_{c^{\prime} x} \cup \Gamma_{c}$ and $R_{3}$ be the region bounded by $\Gamma_{c} \cup C_{c^{\prime} x}$ $\cup C_{a^{\prime} x} \cup \Gamma_{a}$, noting that no point of $R_{2}$ is near $a^{\prime}$ and no point of $R_{3}$ is near $b^{\prime}$.

We consider connected components of * $\left.\mathbb{R}^{2}-\left(S \cup \Gamma_{a} \cup \Gamma_{b} \cup \Gamma_{c}\right)\right)$ that contain standard points (and so are external to $V$ ). These cannot intersect more than one of our three regions. This is because these connected components are bounded by portions of the the outside of $S$ together with portions of the $\Gamma$ curves, and since $S$ does not intersect $Y$, a point traveling along $S$ may only move from one of the three regions into another one by passing through $\Gamma_{a}, \Gamma_{b}$, or $\Gamma_{c}$. The arc $A$ of infinitesimal length can be a part 
of the boundary of only one of these components, since it does not intersect $\overline{B_{a}}, \overline{B_{b}}$, or $\overline{B_{c}}$. Thus, the boundary of all of these components except one is completely contained in ${ }^{*} \mathbb{R}^{2}-{ }^{*} \operatorname{st}\left(Y^{\prime}\right)$.

No standard points of * $s t\left(Y^{\prime}\right)$ can be on any of the boundary points of $R_{1}, R_{2}$, or $R_{3}$, so all of the standard points of this set are in one of the three regions $R_{1}, R_{2}$, or $R_{3}$. We will assume, wlog, that there is a standard point $p_{1}$ in $R_{1}$. Since $p_{1}$ is a standard point it cannot be in $V$, so from above $\mathcal{C}\left(p_{1},{ }^{*} \mathbb{R}^{2}-\left(S \cup \Gamma_{a} \cup \Gamma_{b}\right)\right)$ must be contained in $R_{1}$. But $s t\left(c^{\prime}\right)$ cannot be in $R_{1}$ since no point in $R_{1}$ is within an infinitesimal distance of $c^{\prime}$, and so wlog we suppose that it is in $R_{2}$. By a similar argument, $\mathcal{C}\left(s t\left(c^{\prime}\right),{ }^{*} \mathbb{R}^{2}-(S \cup\right.$ $\left.\Gamma_{b} \cup \Gamma_{c}\right)$ ) is contained in $R_{2}$. But from above, at least one of these sets is contained in a connected component of * $\left.\mathbb{R}^{2}-\left(S \cup \Gamma_{a} \cup \Gamma_{b} \cup \Gamma_{c}\right)\right)$ whose boundary lies in the complement of ${ }^{*} s t\left(Y^{\prime}\right)$. This contradicts the connectedness of ${ }^{*} s t\left(Y^{\prime}\right)$, finishing the proof.

It is easy to see that the condition that $\bar{V}$ contains no standard points is necessary, for without it we could let $Y$ be the set in the nonstandard plane consisting of the closed line segments from $(0,1)$ to $(0,-1)$ and $(0,0)$ to $(1,0)$, and let $V$ be the interior of a simple closed curve that traces around $Y$, and stays infinitesimally close to it at all points. Such a $V$ satisfies the other conditions of the proposition (the boundary of $V$ does not intersect ${ }^{*} s t(V)$ at all), but certainly contains the $\mathrm{Y}$-set that we surrounded, as shown in figure 2 .

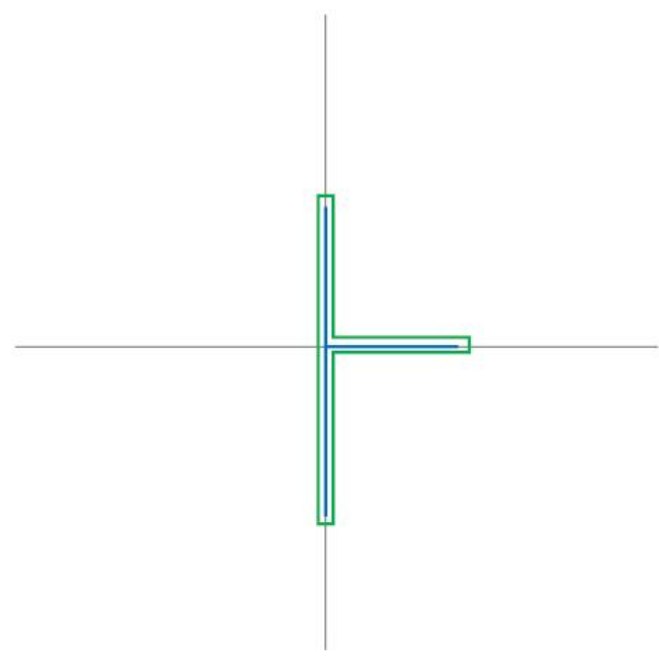

Figure 2: The region must contain no standard points 
Similarly we can see that we cannot allow the curve to intersect the standard part on more than one small arc. If we move the example above up and over by an infinitesimal distance we can surround it with a simple closed curve containing no standard points if we allow the curve to have two disjoint arcs of infinitesimal length intersect the standard part, rather than only one, as in the theorem. In this case the two disjoint arcs can even be infinitesimally close to each other (though not close as one follows along the curve). This is illustrated in figure 3 .

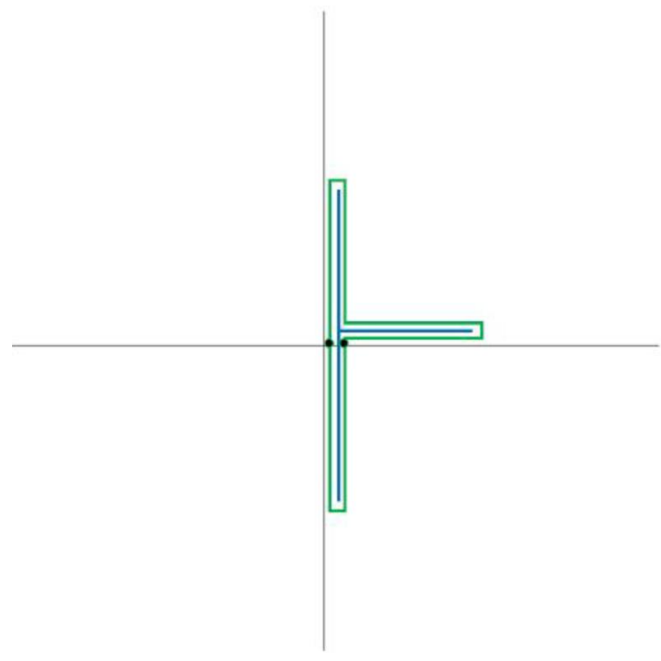

Figure 3: The region must intersect its standard part only in a small arc

It seems unlikely that the condition that $s t(V)$ does not disconnect the plane is necessary in the main result above, but no easy way to remove this condition in the proof is apparent. As is discussed more in section 4, the study of planar continua that do not separate the plane is the fundamental motivation for this work, and in that context this condition will always hold. Still, it would be nice to know if it can be removed from the hypotheses in the result above. It would be easy to see that we could remove this condition if every Y-set whose standard part disconnects the plane contains a Y-set whose standard part does not disconnect the plane, but there are counterexamples to this. One such example takes the union of the following three sets: the closed interval from $(-1,0)$ to $(0,0)$, the graph of the function $y=x \sin (1 / x)$ on $(0,1]$ and the line segment from $(0,0)$ to $(1,1+\zeta)$ where $\zeta$ is infinitesimal.

The theorem below is a standard translation of theorem 2.1. 
Theorem 2.2 Let $E$ be a non-separating compact set in the plane, and $R_{n}$ be a sequence of regions bounded by simple closed curves $S_{n}$ with the property that for any $k$ and any finite collection of points in the plane $\left\{p_{1}, p_{2}, p_{3} \ldots p_{k}\right\}$ there exists an $n$ such that $\left\{p_{1}, p_{2}, p_{3}, \ldots, p_{k}\right\} \cap \bar{R}_{n}=\varnothing$ and $E \cap S_{n} \subset A_{n}$, where $A_{n}$ is an arc of length less than $1 / k$ and all points of $S_{n}$ are within $1 / k$ of some point in $E$. Then for all $\delta>0$ there exists an $n$ such that $R_{n}$ contains no size $\delta$ Y set.

Proof We suppose that there exists some $\delta>0$ for which no such $n$ exists. We consider the collection of mathematical statements " $\{R$ is a region with simple closed curve boundary $S,\left\{p_{1}, p_{2}, p_{3}, \ldots, p_{k}\right\} \cap \bar{R}=\varnothing,{ }^{*} E \cap S \subset A$, where $A$ is an arc of length less than $1 / k$,all points of $S_{n}$ are within $1 / k$ of some point in ${ }^{*} E, R$ contains a size $\delta \mathrm{Y}$ set $\}$, where $\left\{p_{1}, p_{2}, p_{3}, \ldots, p_{k}\right\}$ includes every finite subset of points in the plane. By the hypothesis (and transfer), for any finite subset of this collection there exists an $S_{n}$ and $R_{n}$ that makes all the statements in the finite subcollection true. Thus by $\beth_{1}^{+}$ saturation there exists a single internal region $R$ bounded by an internal simple closed curve $S$ satisfying all the statements at once, and thus satisfying the conditions of the theorem. All of the conditions needed to apply theorem 1 now hold except that it is possible that $s t(V)$ (which must be contained in $E$ ) disconnects the plane. However, since $E$ does not, any point disconnected from infinity by $s t(V)$ must be in $E$, and any point disconnected from infinity and not within an infinitesimal distance of $s t(V)$ is in the interior of $E$. In the proof of theorem 2.1 we use the fact that points inside the original balls satisfying the first four conditions are not disconnected from infinity by $s t(V)$, and that must still be the case. Thus, the conclusion of the theorem holds, and this contradicts the fact that this $V$ contains a size $\delta$ Y-set.

This theorem appears to be significantly stronger than what we obtain by replacing the condition that " $E \cap S_{n} \subset A_{n}$, where $A_{n}$ is an arc of length less than $1 / k$ " with simply " $E \cap S_{n}=\varnothing$ ". Still this weaker result yields the following standard corollary, which is similar to the classic result of Moore's about triods in the plane [13], although here it is well known from Moore's work and "folklore" results that the condition "non-separating" in the corollary below is extraneous.

Corollary 2.3 For every $\delta>0$ no non-separating compact set in the plane can contain infinitely many disjoint connected components each of which contains a size $\delta$ Y-set.

Proof If such a set $E$ existed then given any $m$ distinct components of $E$ each of which contains a size $\delta$ Y set, we may surround these components with non-intersecting simple closed curves all of whose interiors are everywhere within $1 / m$ of $E$ but are 
such that the boundary curves do not intersect $E$. If we focus on a fixed countable set of components of $E$ the collection of all finite subsets is countable, and for each finite subset we obtain finitely many simple closed curves. The countable collection of all of these simple closed curves now forms a collection $\left\{R_{n}\right\}$ that satisfies the conditions of the theorem above (with each $A_{n}$ the empty set). This is because given any finite collection of $k$ points, any subcollection that covers $k+1$ distinct components in the manner described must include one whose closure contains none of the given points, and by construction the other conditions are satisfied. But the conclusion of the theorem contradicts that each of these contains a size $\delta$ Y set.

A note on the strength of these standard results: in a comment on an earlier version of this paper it was pointed out that the corollary above can be derived easily from results in Moore's classic book [14]. It is reasonably likely that the theorem above can be derived from results in that book as well, although probably not as easily, partly due to the fact that, although the result is natural in a nonstandard setting, it is somewhat cumbersome when translated into a standard result. The real strength of the nonstandard result lies not in its standard translation, but in the fact that it allows us to use saturation arguments to seek situations in which internal regions contain no standard points, and thus contain no Y-set. This provides a new nonstandard tool that can be used to approach standard problems in continuum theory.

\section{Examples and Extensions}

The position of the standard points inside an internal region is subtle. For example we consider the usual $\sin (1 / x)$ continuum partially enclosed by an internal simple closed curve as shown in figure 4 . We let $p$ be a point on the curve that is an infinitesimal distance from the $y$ axis and assume that the simple closed curve is "closed off" by a line segment in such a way that $p$ is inside the region. The point $q$ is a standard point on the curve. In this example the standard part of $p$ is outside the curve, whereas the standard parts of all the points that are more than an infinitesimal distance from the axis are all inside the curve. This is true regardless of the width of the region - i.e. even if the distance from the curve to the continuum is a fixed infinitesimal distance. The next result shows why this must be true, and provides important simple conditions under which a region will contain no standard points.

Proposition 3.1 Suppose that $A$ is a standard (closed) arc, $B$ is a standard infinite arc, $a$ and $b$ are two points on ${ }^{*} A$ that are an infinitesimal distance apart and ${ }^{*} B[b, a] \cap$ 


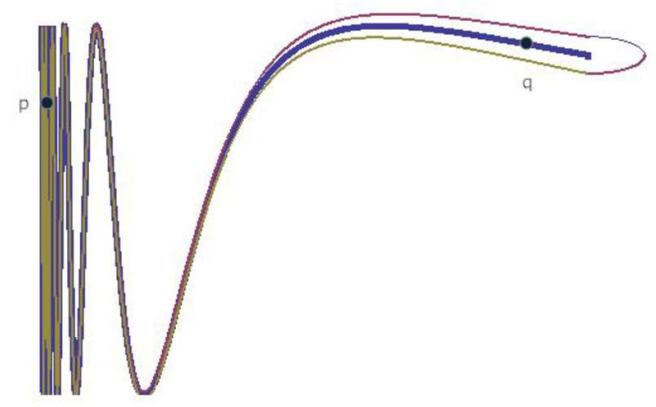

Figure 4: Standard parts move from inside to outside as we move along the curve

${ }^{*} A=\{a, b\}$. Let $S$ be a simple closed curve formed by ${ }^{*} A[b, a]$ together with ${ }^{*} B[a, b]$, and suppose that $s t(a) \notin \overline{V_{S}}$. Then $V_{S}$ contains no standard points (we note that $\operatorname{st}(a)=s t(b))$.

Proof Suppose that $x$ is a standard point in the plane contained in $V_{S}$. Since $\operatorname{st}(a) \notin \overline{V_{S}}$ it is not on ${ }^{*} A[b, a]$. If $\delta>0$ is any noninfinitesimal real number the hypotheses show that in the nonstandard universe there are portions of $B$ that intersect portions of $A$ to form these "loops" in arcs not containing st(a) but such that both endpoints are within $\delta$ of $s t(a)$. By transfer this is also true in the standard universe, which means that for every such loop formed in this way there must be similar ones that are nearer to $s t(a)$. Using the transfer principle again we see that this must be true in the nonstandard universe as well. However no loop formed in this way can be nearer to $\operatorname{st}(a)$ and contain $x$ without intersecting the boundary of $V_{S}$. Since no two points on $B$ intersect, and since the loops only intersect ${ }^{*} A$ at the two endpoints this is not possible.

The example illustrated in figure 4 shows the importance of the condition that ${ }^{*} B[b, a] \cap$ ${ }^{*} A=\{a, b\}$. The boundary of the region in that example could be a portion of a standard infinite arc - one that travels back and forth approaching the sine portion of the curve more closely each time. If $A$ is any standard arc that begins on the y axis and ends at some point with intersection on the continuum we see that all the conditions of the proposition could be satisfied except that the portion of the standard infinite arc in question will always intersect the standard arc in infinitely many places. There are standard arcs that intersect this portion of the curve at exactly two points, as required in the conditions of the proposition, and those portions do, in fact, contain standard 
points, but in those cases the standard part of the endpoint will be contained in $\overline{V_{S}}$. The proposition shows that regardless of the width of the region, the standard parts must stay inside until the curve is within an infinitesimal of the y axis - otherwise we could find a standard arc that intersects the curve at exactly two points and does not contain the standard point of the intersection. But then, by the proposition above, no standard points could be contained in the region.

The conclusion of theorem 2.1 cannot be improved from " $V$ contains no Y-set" to " $V$ is IL-chainable", at least not without additional assumptions. If a set is $\delta$-chainable then it is easy to see that it can contain no size $\delta$ Y-set, so IL-chainability certainly implies that the set contains no Y set. Figure 5 provides an example that shows that the converse is false. If $a$ (the width shown) is infinitesimal and $b$ (the length shown) is not, we see that this figure contains no Y-set, and it is easy to see that it is not IL chainable. This example does not yet show that the conditions given in theorem 2.1 are not strong enough to allow us to conclude IL-chainability, however, as the set shown does not satisfy those conditions. Any simple closed curve containing a set of this shape would either contain standard points or would intersect its standard part on more than a single infinitesimal arc.

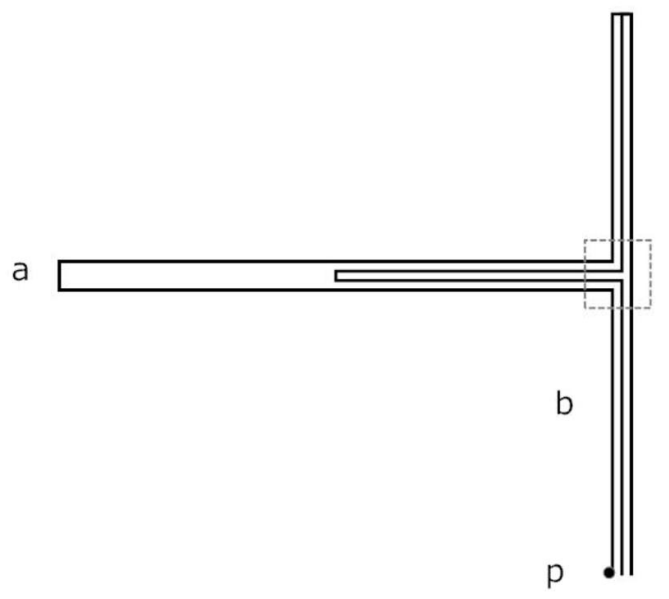

Figure 5: Contains no Y set

In order to see that theorem 2.1 cannot be improved to allow us to conclude ILchainability we consider Ingram's construction of a continuum $A$ that has uncountably many connected components all of positive "span" [7]. Lelek introduced the notion 
of the span of a metric space in 1964 [9] and proved that chainable continua have span zero. See, for example, [16] for related definitions and results.

Definition 3.2 Let $E$ be a continuum with metric $d$, and let $\mathcal{C}$ be the set of all subcontinua $C$ of $E \times E$ with the property that $\pi_{1}(C)=\pi_{2}(C)$. The span of $E$ is

$\sup _{C \in \mathcal{C}} \inf \{d(a, b):(a, b) \in C\}$.

In Ingram's construction there must exist a $\delta>0$ such that infinitely many connected components of $A$ have span greater than $\delta$, and thus cannot be chained with a collection of mesh less than $\delta$. Using a saturation argument similar to that used in the proof of theorem 2.2 we can obtain an example of an internal region $V$ surrounded by an internal simple closed curve $S$ that does not intersect ${ }^{*} A$ at all and contains no standard points but does contain an internal connected component of ${ }^{*} A$ that has internal span greater than $\delta$ (and thus cannot be covered by an internal chain of mesh less than $\delta$ ). All the hypotheses of the theorem are satisfied, but $V$ is not IL-chainable.

Additional conditions that would allow us to conclude that the region is IL-chainable would be highly desirable, and could lead to applications to standard questions involving chainability. One simple condition that is sufficient is that $\operatorname{st}(V)$ itself be chainable. Any chain of standard open sets for $s t(V)$ would also form a chaining set for $V$, so that $V$ could be covered by an internal chaining set of arbitrarily small non-infinitesimal mesh and thus one of infinitesimal mesh. This condition is not very useful, and is far from necessary. An internal "canal" could be IL-chainable and have a standard part that includes a disk simply by winding back and forth and staying within an infinitesimal distance of the previous pass. Finding other conditions that allow us to conclude the chainability of $V$ would be highly desirable. Hoehn has recently shown that there exist non-chainable plane continua that do not have span zero [4], so it would also be interesting to find additional conditions that guarantee that the region must have infinitesimal span.

\section{Fixed Points}

Does every non-separating plane continuum have the fixed point property? This question is usually referred to as the plane fixed point problem. An extensive and comprehensive overview of results and questions related to the plane fixed point problem can be found in [2]. One of the motivations for the results in this paper was to try to find more situations where the classic "dog-chases rabbit" argument (Bing's terminology) 
works to prove results related to the plane fixed point problem. The idea of this type of argument is to move from points toward their images in such a way that the images are "trapped" and chased down by the points until a fixed point is found. The proposition below provides an example of such an argument in this setting.

Proposition 4.1 Let $E$ be a non-separating plane continuum and $f$ be a continuous function from $E$ to $E$. Let $D$ be a connected subset of ${ }^{*} E$ and $R$ a connected, open region containing $D$, and suppose that the following conditions are satisfied:

i) $R$ is IL-chainable, i.e. $R \subset \cup_{k=0}^{n} G_{k}$, with each $G_{k}$ of infinitesimal diameter and $G_{i} \cap G_{j}$ nonempty iff $|i-j| \leq 1$.

ii) The boundary of $R$, except perhaps on $R \cap G_{0}$, is contained in the complement of $E$, i.e. $\partial R-G_{0} \subset{ }^{*} \mathbb{R}^{2}-E$.

iii) There exists an index $j$ and a point $p$ in $D \cap G_{j}$ such that $f(p) \in D-\cup_{k=0}^{j} G_{k}$.

Then $f$ has a fixed point.

Proof We first note that conditions i) and ii) imply that for each $i$, if $p \in\left({ }^{*} E \cap\right.$ $\left.\cup_{k=i+1}^{n} G_{k}\right)-G_{i}$ then $\mathcal{C}\left(p,{ }^{*} E-G_{i}\right) \subset{ }^{*} E \cap \cup_{k=i+1}^{n} G_{k}$. Since $p$ and $f(p)$ are both in the connected set $D$ contained in $R$ they are in the same connected component of ${ }^{*} E$ in $R$. Thus, we may assume that $D$ is this connected component of ${ }^{*} E$ in $R$, for if not we replace $D$ by its connected component and the remainder of the conditions are still satisfied.

We first show that all points in $D \cap G_{j}$ must be mapped into $R-\cup_{k=0}^{j} G_{k}$. If not, let $q \in D \cap G_{j}$ such that $f(q) \notin R-\cup_{k=0}^{j} G_{k}$. Let $\varepsilon>0$ be an infinitesimal less than the distance between ${ }^{*} E \cap\left(R-\cup_{k=0}^{i} G_{k}\right)$ and any point in ${ }^{*} E-\left(R \cap\left(\cup_{k=i}^{n} G_{k}\right)\right.$ ) (for $i=1, \ldots, n)$ (here we use condition ii) to see that such an $\varepsilon$ exists). Now we let $\delta>0$ be the corresponding infinitesimal that exists by the uniform continuity of $f$. Since $D$ is compact it can be covered by a (hyper)finite collection of balls of radius $\delta / 2$, and since $D$ is connected there is a sequence of these $\delta / 2$ balls such that the first ball in the sequence contains $p$, each ball in the sequence intersects the next, and the final ball contains $q$. We now note that if any point in any $G_{i} \cap D$ is mapped to $R-\cup_{k=0}^{i} G_{k}$, then so is every point in $G_{i} \cap D$ that is in the same $\delta / 2$ ball, since no point can move far enough to move out of the region, unless it were to move to $G_{i}$ itself. But no point in $G_{i}$ can map into $G_{i}$ since the diameter is infinitesimal and there are no fixed points. But for the same reason, any point in an intersecting $\delta / 2$ ball must also stay inside $R$ and move to a higher numbered set (again, using the fact that it cannot map 
into a region of the same index). Now by following the chain to the point $q$ we get a contradiction.

Thus, every point in $D \cap G_{j}$ must be mapped into $R-\cup_{k=0}^{j} G_{k}$. But $D \cap G_{j} \cap G_{j+1}$ is not empty, or else we could disconnect $D$ by using $\cup_{k=0}^{j} G_{k}$ and $\cup_{k=j+1}^{n} G_{k}$, so there is a point in $D \cap G_{j+1}$ that maps into $R-\cup_{k=0}^{j} G_{k}$, and since no point in $G_{j+1}$ can map into a point in $G_{j+1}$ there is a point that maps into $R-\cup_{k=0}^{j+1} G_{k}$. Then, reasoning as above we see that all points in $D \cap G_{j+1}$ map into $R-\cup_{k=0}^{j+1} G_{k}$, and continuing in this way we see that for all $m>j$, all points in $D \cap G_{m}$ map into $R-\cup_{k=0}^{m} G_{k}$. Thus all points in $D$ map into $R$, but since $f(D)$ is connected, $D$ is a connected component of ${ }^{*} E$ in $R$, and $f(p) \in D$, we must have that $f(D) \subset D$. But from above this means that for each $m$, all points in $D \cap G_{m}$ map into $D-\cup_{k=0}^{m} G_{k}$. This contradicts that $n$ is (hyper)finite, completing the proof.

This particular argument does not use infinitesimals in a fundamental way. If $E, D$ and $R$ are standard in the proposition above and $R$ is $\delta$-chainable then the conditions allow us to conclude that $f$ has a point that moves a distance less than $\delta$. The condition that $D$ is connected and that $E$ can only intersect at one end of the chain are critical. For example in the diagram below the images can "escape" as we move toward $f(p)$ on the set. Similarly, if the connected component of $p$ does not reach the end of the chain and is not the same as the connected component of $f(p)$ then we have not necessarily created a fixed point trap.

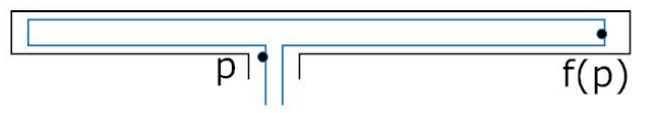

Figure 6: This chainable set has not created a fixed point trap

Returning to the example given in figure 5 we consider the property of containing no Y-sets from the point of view of searching for fixed points. If we imagine this to be a portion of the nonstandard version of a standard continua that connects only at the point $p$ to the rest of the set and $f$ is a standard continuous function from $E$ to $E$ that sends the point $p$ to some other point on the portion shown, then the "dog chases rabbit" argument works here and $f$ must have a fixed point, but the argument is subtle. There are internal (but not standard) continuous functions that could "chase" the image around and let it "escape" without ever getting within an infinitesimal of it, but only if points that are within an infinitesimal of each other are mapped to points that are far apart. This set has noninfinitesimal internal span for a similar reason. 
Under what conditions will a region that contains no Y-set form fixed point traps? Under what additional conditions do sets that satisfy the conditions of theorem 2.1 form fixed point traps? Answers to these questions could provide additional ways to attack unsolved cases of the plane fixed point problem. One of many possible specific conjectures along these lines is given below.

Conjecture 4.2 With $E$ and $f$ as in the fixed point problem let $D$ be a connected subset of ${ }^{*} E$ and $R$ a connected open region whose closure contains $D$ and suppose that the following conditions are satisfied

i) $V$ contains no $Y$-set.

ii) The boundary of $V$, except on an arc of infinitesimal length $A$, is contained in the complement of ${ }^{*} E$.

iii) There exists $p \in D \cap A$ and balls $B$ of arbitrarily small non-infinitesimal diameter about $p$ such that

a) $\mathcal{C}\left(f(p),{ }^{*} E-B\right) \subset V$

b) $V \cap B$ is connected.

c) $V \cap \partial B$ is an arc of infinitesimal length

Then $f$ has a fixed point.

Acknowledgement The author would like to thank the referees for their helpful input and suggestions.

\section{References}

[1] H. Bell, On fixed point properties of plane continua, Trans. Amer. Math. Soc., 128, (1967), 539-548; doi:10.2307/1994475.

[2] C. L. Hagopian, An update on the elusive fixed-point property, in: Open Problems in Topology II, E. Pearl (ed.), Elsevier, (2007), 263-278; doi:10.1016/B978044452208-5/50031-4.

[3] C.W. Henson, Foundations of nonstandard analysis-A gentle introduction to nonstandard extensions, in: Nonstandard Analysis: Theory and Applications, N.J. Cutland, C.W. Henson, and L. Arkeryd (eds.), Kluwer Academic, (1997), $1-50$. 
[4] L. C. Hoehn, A non-chainable plane continuum with span zero, Fund. Math. 211 (2011), no. 2, 149-174; doi: 10.4064/fm211-2-3.

[5] A.E. Hurd and P.A. Loeb, An Introduction to Nonstandard Analysis, Academic Press, 1985.

[6] W.T. Ingram, An atriodic tree-like continuum with positive span, Fund. Math. 77 (1972), 99-107. .

[7] W.T. Ingram, An uncountable collection of mutually exclusive planar, atriodic tree-like continua with positive span, Fund. Math. 85 (1974), 73-78.

[8] W.T. Ingram, A brief historical view of continuum theory, Topology and Its Applications 153 (2006), 1530-1539; doi: 10.1016/j.topol.2004.08.024

[9] A. Lelek, Disjoint mappings and the span of spaces, Fund. Math. 55 (1964), 199-214.

[10] T. Lindstrom, An invitation to nonstandard analysis, in: Nonstandard Analysis And Its Application, N.J. Cutland (ed.), Cambridge University Press, (1988), 1-105; doi: 10.1017/CBO9781139172110.002

[11] P.A. Loeb and M. Wolff, Nonstandard Analysis For the Working Mathematician, Kluwer Academic, 2000; doi: 10.1007/978-94-011-4168-0

[12] J. C. Mayer, Embeddings and prime end structure of chainable continua, Houston J Math. 8 (1982),no. 2, 221-253.

[13] R. L. Moore, Concerning triods in the plane and the junction points of plane continua, Proc. Nat. Acad. Sci., 14 (1928), 85-88; doi:10.1073/pnas.14.1.85

[14] R.L. Moore Foundations of Point Set Theory, rev. ed., American Mathematical Society Colloquium Publications, v. XIII, 1962.

[15] S.B. Nadler, Jr., Continuum Theory, An Introduction, Marcel Dekker, Inc., 1992.

[16] L.G. Oversteegen and E.D. Tymchatyn, On span and chainable continua, Fund. Math. 123 (1984), no. 2, 137-149.

[17] K. Sieklucki, On a class of plane acyclic continua with the fixed point property, Fund. Math. 63 (1968), 257-278.

University of Northern Colorado

steven.leth@unco.edu

http://www . unco.edu

Received: 18 July 2012 Revised: 13 October 2012 